Meta

Journal des traducteurs

Translators' Journal

\title{
Faux sens, contresens, non-sens... un faux débat ?
}

\section{André Dussart}

Volume 50, numéro 1, mars 2005

Enseignement de la traduction dans le monde

Teaching Translation Throughout the World

URI : https://id.erudit.org/iderudit/010661ar

DOI : https://doi.org/10.7202/010661ar

Aller au sommaire du numéro

Éditeur(s)

Les Presses de l'Université de Montréal

ISSN

0026-0452 (imprimé)

1492-1421 (numérique)

Découvrir la revue

Citer cet article

Dussart, A. (2005). Faux sens, contresens, non-sens... un faux débat ? Meta, 50(1), 107-119. https://doi.org/10.7202/010661ar

\section{Résumé de l'article}

L'article décrit les difficultés rencontrées dans l'application des notions de faux sens et de contresens, dont la signification est trop peu différenciée. Un système plus performant d'analyse des traductions est proposé. Il se fonde sur l'évaluation des omissions, des additions et du transfert du sens. La critique tient donc compte des erreurs de traduction mais aussi des réussites, tandis que la triade faux sens/contresens/non-sens n'envisage que les fautes.
Ce document est protégé par la loi sur le droit d'auteur. L'utilisation des services d'Érudit (y compris la reproduction) est assujettie à sa politique d'utilisation que vous pouvez consulter en ligne.

https://apropos.erudit.org/fr/usagers/politique-dutilisation/ 


\title{
Faux sens, contresens, non-sens... un faux débat?
}

\author{
ANDRÉ DUSSART \\ ISTI, Bruxelles, Belgique \\ adussart@heb.be
}

\begin{abstract}
RÉSUMÉ
L'article décrit les difficultés rencontrées dans l'application des notions de faux sens et de contresens, dont la signification est trop peu différenciée. Un système plus performant d'analyse des traductions est proposé. II se fonde sur l'évaluation des omissions, des additions et du transfert du sens. La critique tient donc compte des erreurs de traduction mais aussi des réussites, tandis que la triade faux sens/contresens/non-sens n'envisage que les fautes.
\end{abstract}

\section{ABSTRACT}

This paper analyzes the French concepts of faux sens and contresens, whose meaning is very similar. Their use in translation theory may lead to confusion and should therefore be avoided. Another translation quality assessment system is proposed. It is based on translational practices and discusses the quality of meaning transfer, accuracy and completeness as well as problems of style and language. This system takes both translational errors and successful solutions into account.

\section{MOTS-CLÉS/KEYWORDS}

faux ami, calque, transfert de sens, complétude du sens, précision

L'acquisition d'une construction du réel accompagne l'apprentissage de la langue maternelle, de sorte que les locuteurs s'imprègnent de modèles de pensée et de jugements fondés sur des valeurs propres à une culture, ainsi que l'avaient souligné Von Humboldt, puis, plus près de nous, Leo Weisgerber et les néo-humboldtiens. Le langage influence donc dans une certaine mesure la vision du monde, même s'il convient de se garder d'un déterminisme trop strict à la Whorf.

De nombreuses évaluations, corrections et révisions de traductions s'inspirent d'une triade propre à la langue française: faux sens, contresens, non-sens. D'autres langues, l'anglais et l'allemand, ne connaissent pas tous les termes de cette série. Ainsi, l'ouvrage de Brian Mossop (2001, XVI) consacré à la révision utilise les mots mistranslation et error: "The big problem with using texts in their natural state is that they will most often contain a wide variety of problems: punctuation errors, idiom errors, poor sentence connectors, mistranslations, errors in level of language and so on.» Il semble donc que la distinction faux sens-contresens n'existe pas pour les traductologues et les réviseurs anglophones ou rédigeant en anglais, qui préfèrent parler de mistranslation, d'incorrect translation, d'error, de mistake et de shift (Catford, Popovič). De plus, la triade faux sens/contresens/non-sens suggère une hiérarchisation des fautes: du faux sens, décalage relativement bénin, à l'absurdité, en passant par le contresens en contradiction avec la pensée de l'auteur ou la réalité. 
Avant de juger de l'utilité de cette segmentation, il est nécessaire de se pencher sur les différents éléments de la triade.

\section{Le faux sens: falsche Bedeutung, incorrect meaning (d'après Delisle et al. 1999)}

L'expression la plus ambiguë de la triade est certainement le faux sens, auquel divers auteurs attribuent une signification assez vague et souvent peu compatible avec d'autres opinions rencontrées: "Dans la traduction, un faux sens existe quand la traduction n'est pas juste. ${ }^{\star}$ John loves Mary/John ne déteste pas Mary $\rightarrow$ Jean aime Mary. Un contresens serait: ${ }^{\star}$ John loves Mary $\rightarrow$ John déteste Mary» (www.fhis.ubc. $\mathrm{ca} /$ winder/me/correct.htm). Il est assez curieux que la litote (loves = ne déteste pas) servant d'illustration rappelle le célèbre «Va, je ne te hais point» de Chimène à Rodrigue. L'exemple, qui se veut didactique, paraît malgré tout assez peu vraisemblable, même si des traducteurs littéraires estiment à l'occasion devoir employer une litote non attestée dans l'œuvre originale. À la limite, on pourrait même se demander si cette litote n'est pas davantage une rupture de style qu'une véritable erreur de sens. Par ailleurs, l'interprétation de l'adjectif polysémique juste est assez délicate à formuler. D'après Auroux (1990, tome1: 1406), juste «se dit, également, du résultat de certaines opérations, en tant qu'elles sont conformes aux règles qui président à leur définition ou à leur effectuation (un raisonnement juste; syn. correct)». Selon Lalande $\left(1988^{16}\right.$ : 550), juste équivaut notamment à right, richtig et qualifie «ce qui est exact, correct, rigoureux, précis ». L'ambiguïté transparaît dans les différences notables entre correct et précis: un traducteur peut remettre un texte correct mais manquant de précision, notamment dans la terminologie. Cette indifférenciation due à la polysémie de l'adjectif juste n'est pas trop gênante, mais la définition soulève une objection de taille: comme le contresens n'est pas juste non plus, il est quasi impossible de le distinguer ainsi du faux sens.

Le commentaire suivant ajoute d'autres critères d'évaluation : «Le faux sens consiste à prendre un mot pour un autre. Il peut rester dans le même domaine lexical (maison = mansion, là où on attend house dans le texte) ou changer totalement de catégorie (foyer = home, là où on attend hearth). La faute sera donc plus ou moins grande.» On pourrait discuter de l'importance relative de la faute dans les traductions mansion et home. Des réviseurs ou correcteurs peinent souvent pour s'entendre sur la gravité des fautes, l'expérience l'a maintes fois prouvé. Sans entrer dans cette discussion à propos de l'importance relative des erreurs, on remarquera que le faux sens résulterait donc dans ce cas-ci d'une confusion entre deux mots.

Pour un autre auteur, le faux sens est une «mauvaise appréciation du sens d'un mot ou d'un énoncé dans un contexte donné».

L'auteur souligne que la critique d'une traduction ne se limite pas aux mots; il intègre le niveau des énoncés et donc la dimension textuelle dans son point de vue.

Selon Delisle et al. (1999: 40), il s'agit d'une «faute de traduction qui consiste à attribuer à un mot ou à une expression du texte de départ une acception erronée qui altère le sens du texte, sans pour autant conduire à un contresens [...] Le faux sens résulte habituellement de l'appréciation erronée de la <signification pertinente> d'un mot. Ce glissement de sens dû à une interprétation fautive conduit généralement à une <impropriété $>$ [...] Le faux sens est une erreur moins grave qu'un contresens 
ou un non-sens, car il ne dénature pas complètement le sens du texte de départ. Néanmoins, la ligne de démarcation entre le faux sens et le contresens est parfois difficile à tracer. » À l'appui de la définition, les auteurs citent: a reasonable amount of stress, ${ }^{*}$ une quantité raisonnable (= une certaine dose de stress); Cancun as a resort is scarcely 10 years old, ${ }^{*}$ Cancun n'est qu'un lieu de villégiature depuis 10 ans (Cancun est un lieu de villégiature depuis 10 ans à peine). Le premier exemple correspond à la définition, tandis que le deuxième présente en fait une erreur syntaxique (scarcely porte sur 10 years) et non une faute lexicale (une erreur d'appréciation du sens de scarcely). Les concepts de référence mentionnés dans l'entrée faux sens sont faux ami, interférence, mot juste, ce qui laisse supposer que les faux amis et les calques font partie des faux sens.

La notion de glissement de sens suggère que le faux sens est un décalage, une déviation, un écart simple par rapport à la réalité textuelle, une équivalence partielle. Dans ce cas, il faudrait ranger les surtraductions et les soustraductions dans les faux sens, à côté des faux amis, des calques. Le faux sens finit par englober beaucoup d'erreurs ou mistranslations. Par ailleurs, la référence à une altération du sens risque d'introduire la confusion, puisque certains traductologues se fondent précisément sur le critère de dénaturation du sens (sinnentstellender Fehler chez Kade) pour définir le contresens.

Voilà un quart de siècle, Ladmiral (1979: 62) avait déjà tenté de distinguer ces termes: «Quant au faux sens, on voit souvent en lui un contre-sens au petit pied [...] D'une façon plus systématique, on pourra distinguer deux grands types de fautes: d'une part la triade non-sens/contre-sens/faux sens, où les fautes sont des erreurs d'interprétation portant sur la signification même du texte, et d'autre part un nuage de fautes plus minimes qui sont des fautes de français, portant sur la structuration terminale du signifiant-cible [...] Le faux sens ressortit à un problème d'expression en français langue-cible maternelle (L1), le contre-sens à la compréhension du textesource...» Il convient d'observer que Ladmiral est le seul traductologue à utiliser la distinction langue de départ - langue d'arrivée (dans sa terminologie : langue source - langue cible), pour définir le faux sens. Si on le suit, le faux sens serait une erreur d'écriture, un mauvais choix lexical en langue d'arrivée, tandis que le contresens serait une erreur d'interprétation (misunderstanding ou misinterpretation) du texte.

Avant d'aller plus loin, il faut se poser une question relative à la signification de faux dans cette expression. Lalande $\left(1988^{16}: 1223\right)$ signale que vrai (wahr, echt, wirklich), l'antonyme de faux, c'est le «caractère de l'assertion à laquelle il est légitime de donner un plein et entier assentiment» et encore «qui existe réellement, par opposition à ce qu'on raconte». Sans entrer dans le débat philosophique à propos de l'opposition vrai-faux, on pourrait en déduire que le faux sens s'oppose donc à un vrai sens auquel il est légitime de se rallier. Est faux ce qui s'oppose au vrai ou, tout au moins, à ce qui est généralement admis comme tel. Si $p$ est rouge, alors il n'est pas vert. Si $q$ est vert, alors il n'est pas rouge. Cette dichotomie s'utilise dans les signaux lumineux de la circulation routière. Or le faux sens tel que décrit jusqu'ici ne semble pas s'inscrire dans cette dualité, puisqu'il n'est pas vraiment en opposition, en contradiction avec le vrai sens, sinon il se confondrait avec le contresens. L'une des acceptions de faux (Rey, 2001, tome 3:644) le confirme: faux est aussi ce "qui marque un écart par rapport à ce qui est correct, normal», de sorte qu'il est impossible d'assigner à faux un sens clair. 
D’après Le Petit Robert (2003), faux signifie « erroné» (qui contient des erreurs), «inexact», à côté d'autres acceptions qui ne concernent pas le faux sens: "artificielfactice, qui n'est pas ce qu'on nomme (faux acacia), injustifié (fausse joie) ». L'expression faux sens est ainsi définie par Le Petit Robert (2003 : 1043) : «Faire un faux sens: interpréter d'une manière erronée le sens d'un mot dans un texte.» Étant donné que Le Larousse de la langue française, Lexis (Dubois, 1979: 1720) définit précisément le contresens comme "l'interprétation erronée d'un mot, d'une phrase», le faux sens se superpose exactement au contresens. Où s'arrête donc le faux sens, où commence le contresens? De l'aveu même de Delisle et al. (1999: 40), la ligne de démarcation est souvent difficile à tracer. Mais alors, quelle est l'utilité de cette notion de faux sens, si elle prête à confusion?

Le Trésor de la langue française (1992, tome 15:322) se contente d'écrire: «Erreur sur la signification d'un mot dans un texte.» Le site <http://www.limsi.fr/Individu/ gs/DLF/fauxSens.html $>$ (Limsi = Laboratoire d'informatique pour la mécanique et les sciences de l'ingénieur) cite un grand nombre d'exemples de faux sens, parmi lesquels: achever ne signifie pas arrêter/cesser; affabulation (trame d'un récit) n'est pas fabulation (substitution de rêves à la réalité), alternative n'est pas solution de rechange; conséquent n'est pas important, etc. On se rapproche de la définition du faux sens donnée par Ladmiral dans le domaine de la traduction. Le faux sens est donc exclusivement l'usage erroné, incorrect, impropre d'un mot, par exemple, lorsqu'un traducteur choisira «conséquent» pour équivalent de «wichtig», «important». Dans ce contexte unilingue, l'expression faux sens se justifie pleinement. Elle est en revanche inadéquate pour qualifier une traduction fautive.

Un test rapide révèle les hésitations des spécialistes vis-à-vis de cette triade. Le verbe allemand fordern (demander, réclamer, exiger) se conjugue [er] forderte au prétérit face à [er] fordert au présent. Si le traducteur fatigué, distrait ou pressé néglige la désinence $e$ de $[e r]$ forderte et transpose l'action au présent, il ne peut s'agir d'un faux sens selon les précédentes définitions axées sur le sens du mot. C’est une erreur d'analyse grammaticale et non un choix défectueux de mots ("prendre un mot pour un autre», "une mauvaise appréciation du sens d'un mot», etc). La confusion entre «fördern» (faire progresser) et «fordern» serait, quant à elle, une erreur lexicale. Dans la confusion "forderte-fordert» (demande-demandait), serions-nous en présence d'un contresens? Si l'on interroge des traducteurs et des enseignants à ce sujet, ils répondent quasi unanimement par la négative. Comme cette faute n'est pas non plus un non-sens, où est donc l'erreur?

Les deux traductions suivantes semblent contenir des faux sens. Mais est-on sûr que chaque observateur les considérerait comme telles dans ce travail de fin d'études (traduction partielle du livre de Bill Jones et Dennis Kavanagh (1998), British Politics Today, Manchester U.P, ch. XVII) :

Shorn of Empire and economic preeminence, the country indulged in gloomy introspection ${ }^{*}$ Dépossédé de son Empire et de sa prépondérance économique, le Royaume-Uni sombra dans une profonde introspection (gloomy = profond ? indulged = sombrer?)

... the need for the production of goods, which in turn creates employment

... ${ }^{*}$ la production de biens devient nécessaire, ce qui, à terme, crée de l'emploi (in turn = à terme?) 
Analysons un autre exemple de choix erroné d'un terme:

... die wohlbeleutchtete Halle, an deren linker Seite Gesellschaftsräume lagen (Thomas Mann [1975], Der Zauberberg, Frankfurt a. M.: Fischer Taschenbücher, p. 14):

... ${ }^{*}$ le hall bien éclairé, à droite (?) duquel se trouvaient des salons (trad. M. Betz [1989], La montagne magique, Paris, Le livre de poche, p. 19).

La faute de distraction (linker Seite/à droite) est bénigne et elle ne gêne nullement le déroulement de l'histoire. Il en irait différemment dans un texte technique. S'agit-il d'un faux sens? Oui, si l'on applique les critères cités: «la traduction n'est pas juste»; «le faux sens consiste à prendre un mot pour un autre»; c'est « une mauvaise appréciation d'un mot». Ou bien sommes-nous plutôt en présence d'un contresens? Oui, si l'on envisage l'opposition gauche-droite, donc la contradiction. Comment décider objectivement d'après le commentaire: le «faux sens altère le sens du texte, sans conduire au contresens» (Delisle et al., 1999: 40)?

Bien plus que la gravité d'une erreur, notion très relative et trop liée à des jugements personnels, et qui, par ailleurs, ne s'évalue pas uniquement par rapport à la grammaire ou au lexique, mais principalement dans le texte, la contradiction avec le savoir, la logique ou simplement le sens commun peut servir de critère objectif. De ce point de vue, il semblerait plus aisé de s'accorder sur le contresens, si le concept de faux sens ne venait pas brouiller les cartes.

Un exemple plus prégnant de faute absurde, apparemment un simple faux sens, est analysé par Milan Kundera (1993: 123); l'écrivain, fâché avec les traducteurs, critique la transposition d'une phrase de Kafka dans Le Château:

«Dort vergingen Stunden, Stunden gemeinsamen Atems, gemeinsamen Herzschlags, Stunden in denen K immerfort das Gefühl hatte, er verirre sich oder er sei so weit in der Fremde, wie vor ihm noch kein Mensch, einer Fremde, in der selbst die Luft keinen Bestandteil der Heimatluft habe, in der man vor Fremdheit ersticken müsse und in deren unsinnigen Verlockungen man doch nichts tun könne als weiter gehen, weiter sich verirren.»

Kundera commente la traduction de "gehen » par «marcher» au lieu de «aller» chez Vialatte. Selon les définitions analysées ci-dessus, il s'agit d'un faux sens banal. Et pourtant, comme le fait remarquer Kundera, si on lit attentivement le contexte "gemeinsamen Atems, gemeinsamen Herzschlags" (haleine commune, battements de cours communs), "in deren unsinnigen Verlockungen man doch nichts tun könne als weiter gehen, weiter sich verirren» («où l'on ne pouvait rien faire au milieu de séductions insensées, que continuer à aller, que continuer à s'égarer»), Kafka évoque une relation intime. La description d'ébats amoureux devient absurde et grotesque, si l'on traduit «gehen» par «marcher», comme Vialatte: "et où l'on ne pouvait plus rien faire, au milieu d'insanes séductions, que continuer à marcher, que continuer à se perdre.» Remémorons-nous la chanson de Serge Gainsbourg et Jane Birkin: «Et je vais et je viens entre tes reins»! Le mauvais choix du mot résulte manifestement d'une mauvaise interprétation, qui revient à ne lire dans le texte de Kafka qu'une promenade, cœurs battant à l'unisson, dans une contrée étrange pleine de séductions.

En résumé, pour certains auteurs, faire un faux sens, c'est prendre un mot pour un autre, mal apprécier le sens d'un mot, interpréter le sens de manière erronée, choisir une acception erronée. Ils n'ajoutent pas que l'erreur d'analyse grammaticale ou syntaxique peut donner un faux sens; un seul d'entre eux étend la notion de faux 
sens à l'énoncé et semble donc prendre le niveau syntaxique et textuel en compte. Si l'on convient de classer dans la catégorie des faux sens les faux amis ( «mot [...] dont la morphologie ressemble à celle d'un mot d'une autre langue, mais dont la signification [...] est différente », Delisle et al. 1999: 39), on se heurte à un problème sérieux. En effet, ces faux amis constituent bien souvent de véritables contresens: traduire l'allemand «Infusion» par «*infusion» au lieu de «perfusion» et «Hypertoniker» par « ${ }^{\star}$ hypertonique» (au lieu de «hypertendu»), c'est contredire la science du médecin. Et il serait possible de multiplier les exemples, car les traducteurs chevronnés savent parfaitement que la langue médicale allemande recèle bien des pièges pour le francophone. Donc la graduation entre faux sens et contresens, apparemment si convaincante, n'est plus soutenable. Où se situe la limite entre une acception erronée (Delisle et al. 1999: 40) et un véritable contresens?

À l'instar de Ladmiral, il vaudrait mieux envisager dans un premier temps séparément la restitution du sens et la formulation en langue d'arrivée, même si l'appréciation finale réunit les deux facteurs et même si une expression maladroite ou inadaptée peut masquer ou modifier le sens du texte.

\section{Le contresens: Sinnwidrigkeit, sinnentstellender Fehler (= faute altérant ou dénaturant le sens), mistranslation (caused by a misunderstanding), negative shift; (Delisle et al.: Sinnverkehrung, misinterpretation)}

La notion de contresens paraît plus claire: «[...] comme l'indique son nom, le contresens aboutit à une traduction contraire de ce qui a été énoncé.» L'exemple cité est «il veut conclure la paix» traduit par « ${ }^{\star}$ he wants to wage war» (<http: //www.llsh. univ-savoie.fr/lea/Perdrieau/conseils.htm $>$, encore un exemple didactique peu crédible dans le monde de la traduction).

Selon Delisle et al. (1999: 23), c'est une «faute de traduction qui consiste à attribuer à un segment du texte de départ un sens contraire à celui qu'a voulu exprimer l'auteur ». L'opposition, l'antinomie ou la contradiction est une exclusion logique de termes (links s'oppose à rechts, comme gauche à droite) ou d'énoncés. Il reste à définir la contradiction. Selon Auroux (1990, tome 1:468-469), en logique, «la contradiction, [...] est la conjonction d'un énoncé et de sa négation». Cette faute se rencontre également dans les traductions, elle provoque l'incohérence du discours. Plus loin, Auroux ajoute: "Par extension, on dénomme également contradiction un énoncé, qui, pris en lui-même, est nécessairement faux.» Cette extension renvoie à l'opposition du vrai et du faux et donc indirectement au faux sens... tout cela dans la perspective d'une logique binaire, qui n'est pas applicable en toutes circonstances.

La formulation suivante ne fournit pas davantage de critères distinctifs pertinents: « [...] attribuer à un mot ou à un groupe de mots un sens erroné, ou de façon plus générale, à (!) trahir la pensée de l'auteur du TD». (La première partie de la phrase est une définition déjà rencontrée du faux sens, qui est insuffisamment complétée par la référence à la trahison de la pensée. Certains faux sens trahissent tout autant la pensée de l'auteur; les exemples abondent pour le prouver.)

L'équivalent anglais de contresens est mistranslation ou negative shift. Shuttleworth (1999: 112) écrit: «Negative shift/Defined by Popovič as an incorrect translational solution (or mistranslation) caused by a misunderstanding on the part of the translator». Kade (1980: 91) définit une «sinnentstellender Fehler» comme l'altération 
(Verletzung) du dénotat de l'original dans la traduction. Pour Le Petit Robert (2003: 538), le contresens est une «interprétation contraire à la signification véritable. Faire un contresens et des faux sens dans une traduction». Mais contraire à la signification véritable, c'est aussi la définition de nécessairement faux (voir Auroux), de sorte que l'on s'enferme dans un cercle infernal et que le faux sens ressemble fort au contresens, d'où l'embarras des praticiens pour les distinguer l'un de l'autre. («Néanmoins, la ligne de démarcation entre le faux sens et le contresens est parfois difficile à tracer », selon Delisle et al. 1999: 40.)

Le contresens n'est pas seulement contraire à l'opinion de l'auteur, il s'oppose aux faits de l'expérience et à la logique, de sorte qu'il aboutit parfois à l'impossible ou à l'absurde, donc au non-sens. Un exemple de contresens tiré du manuel d'instruction d'un caméscope Sony DCR-PC8E illustrera ces propos:

It is easier to focus on the subject if you adjust the zoom to shoot at the ' $W$ ' (wide-angle) after focussing at the ' $T$ ' (telephoto) position.

Fokussieren Sie im Telebereich (' $T$ '), nehmen Sie dann aber im Weitwinkelbereich ('W') auf.

${ }^{*}$ Il est plus facile d'obtenir une image nette en mettant d'abord le zoom en position ' $W$ ' (grand angle), puis en faisant la mise au point en position ' $T$ ' (téléobjectif).

La version française du texte est un contresens: pour un photographe, un cinéaste averti ou professionnel, c'est exactement l'inverse qu'il convient de faire. En réalité, comme la profondeur de champ est très faible pour une longue focale, la mise au point précise se contrôle plus aisément dans le viseur en position de téléobjectif, tandis qu'en position de grand angle, la zone de netteté bien plus étendue ne garantit pas une mise au point ultrafine. Par conséquent, tous les photographes ou vidéastes avisés savent qu'il faut d'abord effectuer la mise au point en position de téléobjectif extrême, puis recadrer l'image en choisissant la focale idéale, donc éventuellement une longueur focale moins élevée. Le texte allemand est parfaitement correct: «Faites la mise au point en position de téléobjectif, mais réalisez ensuite la prise de vues en position de grand angle» (traduction de l'auteur). Un lecteur non averti n'y verra que du feu, sauf s'il essaie un jour d'utiliser un caméscope; un utilisateur moyen se posera certainement des questions; un connaisseur sera interloqué et il ornera la marge du texte d'un point d'interrogation.

\section{Le non-sens: Unsinn, Nichtinterpretierbarkeit; Widersinn, nonsense}

La définition du non-sens n'est pas aussi immédiate et intuitive qu'on pourrait le croire. Lalande $\left(1988^{16}: 11\right)$ déclare: «Strictement parlant, l'absurde doit être distingué du non-sens (D. Unsinn, sinnlos); car l'absurde a un sens et est faux, tandis que le non-sens n'est proprement ni vrai, ni faux». Delisle et al. (1999: 58) donnent sinnentstellende Übersetzung pour équivalent de non-sens, ce qui est excessif. Le grand dictionnaire Duden en huit volumes (Drosdowski: 1993) définit entstellen par «im Sinn verfälschen, falsch darstellen» (= fausser le sens). D'après Kade (1980: 90), les termes Unsinn et Widersinn portent un jugement sur l'interprétabilité du texte: certains passages de textes ne sont tout simplement pas compréhensibles. Le non-sens aurait par conséquent au moins deux acceptions, dont l'une serait abusive, si l'on en croit Lalande. Dans cette perspective, il est à noter que Lalande $\left(1988^{16}: 12\right)$ donne Widersinnigkeit pour équivalent de nonsense sous l'entrée absurdité (par opposition à Unsinn). On est en droit de soutenir que l'absurde heurte la raison, l'expérience et le 
sens commun, tandis que le vrai non-sens est ininterprétable. Auroux (1990: 13) illustre le non-sens ininterprétable par les mots « rond un où » et le non-sens absurde par l'expression « un carré rond». Dans les traductions, les deux types de non-sens se rencontrent: la phrase ou le passage illisible, incompréhensible et le texte absurde défiant la logique ou l'expérience. Même le concept de non-sens se discute parfois: tout énoncé apparemment illogique peut avoir un sens pour certains locuteurs seulement (les messages cryptés) ou pour des lecteurs éclairés (la poésie).

Delisle et al. (1999: 58) restreignent le concept de non-sens: «Faute de traduction» qui consiste à attribuer à un segment du texte de départ un sens erroné qui a pour effet d'introduire dans le TA une formulation absurde.» Mossop (2001: 105) en analyse les causes: "The source text makes sense but the translator has introduced nonsense. Among student trainees, such nonsense often arises from lack of sourcelanguage knowledge; among experienced translators, it arises from attention waning when rushed or tired.» Il faut toutefois ajouter que le «nonsense» ne découle pas seulement d'une connaissance imparfaite de la langue source, mais plus souvent d'une maîtrise insuffisante des domaines de spécialité et qu'il s'observe chez des traducteurs s'exprimant très bien à la fois dans la langue de départ et dans la langue d'arrivée.

Le non-sens n'est pas une catégorie à part, il caractérise certaines fautes de traduction (de British Politics Today):

[Margaret Thatcher] She always claimed that she would need two terms in office for her policies to have full effect, and of course ended up serving for eleven and a half years in Downing Street.

Elle qui prétendait constamment avoir besoin de deux mandats pour que ses politiques puissent être pleinement effectives, occupa finalement le 10, Downing Street durant onze ans et demi (ended up serving?).

Et sur la même page, on lit une erreur créant une incohérence:

Margaret Thatcher set the tone for this eighteen-year period by insisting on a rigidly strict monetarist policy.

${ }^{*}$ Margaret Thatcher annonça la couleur de la politique qu'elle mena durant 18 ans...???

\section{Modèle d'analyse de traductions}

Les considérations précédentes ont montré les déficiences et surtout l'imprécision de cette triade. Le faux sens généralement perçu comme l'interprétation erronée du sens d'un mot et parfois d'un énoncé est une notion vraiment trop vague, par rapport au contresens. Au premier abord, le contresens est nettement mieux défini dans sa contradiction avec le savoir, la logique ou l'expérience humaine. Quant au non-sens absurde, il ne peut figurer une catégorie à part entière, il n'est qu'un aspect de certaines erreurs de traduction. Le non-sens indéchiffrable est souvent confondu par les critiques ou les correcteurs d'épreuves avec les négligences stylistiques et les fautes de langue: le charabia et le galimatias ne reflètent pas seulement des fautes de syntaxe et de grammaire, mais bien plus une incompréhension du texte de départ, fréquente chez des débutants.

Pour évaluer des traductions, il serait donc préférable de choisir les critères selon une autre grille, qui permet aussi d'envisager les côtés positifs de la traduction. L'essence même de cette dernière est la substitution d'un texte en langue d'arrivée à un 
original. Le concept de substitution pourrait susciter la critique : en effet, il pourrait laisser entrevoir une définition sous-jacente de la traduction comme un simple échange de mots et de structures, voire comme un littéralisme. Dans les faits, pourtant, le texte d'arrivée doit fonctionner seul dans la communauté linguistique, en l'absence de l'original. Il se substitue à ce dernier. Cette substitution doit assurer le transfert du sens.

Toute confrontation entre le texte de départ et le texte d'arrivée fait apparaître les qualités ou les déficiences aux niveaux dénotatif et connotatif. Les erreurs fréquentes sont bien connues des traducteurs, des réviseurs et des enseignants. Mossop (2001: 5) les cite: "Also, some degree of revision is needed even with ephemeral texts to correct errors which are peculiar to translational writing: mistranslations, omissions, and the strange unidiomatic language which is so hard to avoid when translating (odd word combinations or sentence structures calqued from the source text). » Ce sont donc les fautes de traduction (mistranslations), les calques structurels et l'expression non idiomatique qui constituent les principaux défauts à corriger.

Sur la base de nombreuses constatations, il est, par conséquent, possible de mettre en évidence les mêmes phénomènes récurrents: le traducteur supprime à tort ou à raison des éléments textuels (omissions), parfois il en rajoute (additions). Il effectue des transferts de sens corrects ou incorrects, la cohésion du texte est forte ou faible (cohésion lexico-syntaxique). Enfin, le style et la langue sont à envisager. Les différents aspects évoqués ici prendront plus ou moins d'importance selon le type d'écrit. Tout texte doit être facilement lisible, mais on admettra plus facilement quelques lourdeurs de style dans un mode d'emploi que dans un roman. Par ailleurs, si les erreurs de faits et de logique ne posent en général aucun problème de consensus chez les réviseurs, la critique et la correction du style relèvent d'un jugement de valeur esthétique, donc souvent contestable.

En bref, le schéma suivant est plus opérationnel, plus productif et moins discutable que la triade faux sens, contresens, non-sens:

1- Transfert partiel ou omission

2- Surtransfert ou addition

3- Transfert de sens imparfait (incorrect, imprécis)

4- Cohésion du texte

5- Langue et style

\subsection{Le transfert partiel ou omission}

La traduction partielle est relativement fréquente. Le traducteur doit essayer de restituer tout le sens, mais rien que le sens. Les omissions permettent d'éviter des redondances et des lourdeurs. Malblanc (1968: 267-274) souligne que les traducteurs ne rendent pas toujours les auxiliaires modaux allemands können, mögen, sollen: «Nichts kann den Wein ersetzen/Rien ne remplace le vin». Il existe bien d'autres exemples d'omissions justifiées, mais le traducteur distrait, pressé ou fatigué peut tout simplement oublier un élément ou un groupe de mots. Dans certains passages redoutables, on soupçonnera même un oubli volontaire destiné à escamoter une difficulté d'interprétation ou de rédaction. Parfois, le commanditaire ou la maison d'édition exige l'abandon de certains passages, ou encore des normes techniques différentes d'un pays à l'autre nécessitent la suppression pure et simple. Supposons la 
traduction en français d'un livre allemand décrivant la couverture des toits (cas vécu) : dans les pays à climat chaud et sec, le chaume est interdit, de sorte qu'il faudra éliminer une partie du texte (avec l'accord des détenteurs de droits).

\subsection{Le surtransfert ou addition}

Les additions sous forme de paraphrases, de gloses, de compensations et de renforcements se rencontrent fréquemment. Les traductologues ont maintes fois constaté que les traducteurs littéraires ont tendance à expliciter le texte, dans le but, semble-t-il, d'amener le lecteur du texte d'arrivée à mieux en saisir le sens:

Ces salles étaient d'ailleurs équipées pour soigner les malades dans le minimum de temps. These wards, it was explained, were equipped to provide patients with immediate treatement (d'après Guillemin-Flescher, 1988: 15).

\subsection{Le transfert imparfait du sens}

Pour qu'un texte traduit puisse se substituer parfaitement à l'original, il faut que le transfert de sens soit correct (a), précis (b) et complet (c), en adéquation avec la situation et le contexte. Les fautes de sens ou incorrections (a) sont dues à des lacunes dans la compétence en langue de départ (du reste, qui a une compétence parfaite même dans sa langue maternelle, en dehors du locuteur idéal postulé par des grammairiens?), à une ignorance dans un domaine du savoir ou du comportement en société et à des illogismes. Le contresens traditionnel recouvre bien ce genre d'erreurs. La précision (b), conçue comme un écart minimal, voire nul, entre le sens du texte de départ et du texte d'arrivée, est une deuxième exigence de la bonne traduction. La précision s'oppose à l'approximation pouvant mener à des conflits d'interprétation, notamment dans les textes juridiques. Un exemple désormais célèbre est le segment de phrase ambigu: "to retire from occupied countries», qui peut se traduire "se retirer de [ou des?] territoires occupés ».

La complétude (c) du sens contribue au respect strict de la pensée d'un auteur. La sous-traduction résulte de la perte partielle du sens originel au cours du transfert. Selon Newmark, cité par Shuttleworth (1999: 191), la perte de sens inévitable dans toute traduction se manifeste soit par un degré supérieur de spécification, soit par une plus grande généralisation du sens par rapport à l'original. Un plus haut degré de généralisation définit ainsi la sous-traduction (undertranslation), tandis que la surtraduction (overtranslation) est une spécification supplémentaire du sens. D'autres définitions moins valables existent, dont celle de Vinay-Darbelnet (1978: 32): «La surtraduction consiste essentiellement à voir deux unités là où il n'y en a qu'une ». Si les contenus sémantiques des équivalents en langue d'arrivée ne sont pas parfaitement «symétriques» au sens des termes de l'original, le traducteur devra contrebalancer la perte en utilisant, par exemple, la compensation. Bien souvent également, l'incomplétude du sens résulte d'une maladresse du traducteur et non d'une «insuffisance» de la langue d'arrivée. On pourrait regrouper les imprécisions et l'incomplétude du sens dans la catégorie plus générale des glissements de sens. 


\subsection{La cohésion du texte}

La cohésion du texte est assurée par les liens, les chaînons du discours (les conjonctions et les adverbes notamment), souvent difficiles à traduire, mais aussi par les parallélismes de constructions syntaxiques, les démonstratifs, les pronoms, les récurrences de lexèmes ou de syntagmes de signification très proche. Il n'est pas possible d'envisager ici en détail tous ces aspects de la cohésion du texte. On se contentera de rappeler que les traducteurs ne suivent pas toujours l'original dans ses répétitions, sans doute parce que l'école leur a inculqué la nécessité de varier le vocabulaire. Or, ces répétitions et les récurrences facilitent l'interprétation du texte, alors que des synonymes ou des paraphrases provoquent des ruptures qui peuvent dérouter le lecteur.

Si les aspects précédemment évoqués de la cohésion sont bien connus, il n’en va peut-être pas de même pour les temps du discours, marqués de différentes façons et notamment par les verbes dans un certain nombre de langues. Le « je» du locuteur se situe dans un lieu ( $h i c$ ) et un temps linguistique (nunc). Le temps linguistique (différent du temps physique et du temps chronique, Benveniste 1974: 70) contribue au repérage énonciatif et, par conséquent, à l'ordonnancement du discours. La coïncidence ou la contemporanéité de certains faits et événements s'oppose au prospectif (le futur, dénommé prospection par Benveniste) et au rétrospectif (le passé) du discours. Il importe que le texte traduit restitue correctement ces jalons temporels reliés entre eux au sein du texte. Or, il est fréquent que les traducteurs transforment ces repères (par négligence ou hâte excessive?) et gomment ainsi les interconnexions du texte.

\subsection{La langue et le style}

Il n'est guère nécessaire d'insister longuement sur les aspects stylistiques, sur le respect du niveau ou registre du discours, sur l'emploi correct des technolectes, ni sur l'observation stricte des règles de grammaire, d'orthographe et de ponctuation. Mossop (2001: 101) croit voir une opposition entre la recherche d'une précision absolue et la qualité du style et, par conséquent, une contrainte pour le traducteur: «At the higher levels of precision, a degree of readability is inevitably sacrificed, while at the higher levels of reader-friendliness, accuracy must suffer ». Il ne faut toutefois pas surestimer les difficultés issues de ces exigences contradictoires. La traduction implique des stratégies et des arbitrages, compte tenu de la situation de la communication et du public-cible.

Des calques, en apparence assez anodins, expliquent bien des maladresses dans l'expression. Tout traducteur a tendance à s'inspirer inconsciemment de l'original et il est susceptible de commettre des erreurs d'usage et d'idiomaticité, même s'il écrit naturellement bien dans sa langue maternelle:

Dies lässt sich gut in lokaler Betäubung und ambulant durchführen

${ }^{*}$ L'opération est praticable sous anesthésie locale et de façon ambulatoire (une opération praticable de façon ambulatoire est pour le moins curieuse!)

Au lieu de: L'opération est facilement praticable sous anesthésie locale et en médecinel traitement ambulatoire. 
Sie [Tänzer] erhalten zwar ihre monatliche Gage

${ }^{*}$ Bien sûr, ils [les danseurs] reçoivent leur gage mensuel

(Le gage est une garantie - mettre sa montre en gage, un gage de fidélité - ou une pénitence au jeu, tandis que les gages sont des appointements).

\section{Conclusion}

Cette triade faux sens/contresens/non-sens laisse perplexe, car le seul élément aux contours nettement définis est le contresens. Dès que ce dernier est confronté au faux sens, l'hésitation et l'indécision s'installent. Pour la plupart des auteurs, le faux sens ne concerne que des mots à l'exclusion des énoncés, de sorte que la dimension syntagmatique n'est absolument pas prise en compte. De plus, l'évaluation du transfert de sens ne se fonde pas uniquement sur la signification des mots, mais aussi sur une analyse du texte et de la situation du discours. Toutefois, le faux sens se justifie pleinement pour qualifier l'usage impropre d'un terme; l'erreur est de l'appliquer à la transposition de textes dans une autre langue. Par ailleurs, le non-sens peut caractériser aussi bien certaines erreurs de traduction. Le non-sens absurde implique nécessairement un contresens ou un faux sens, mais l'inverse n'est pas nécessairement vrai.

D'autres modèles de critique et d'analyse sont plus performants. L'expérience des traducteurs et des réviseurs sert de fondement au schéma envisagé. Ce dernier a au moins le mérite de ne pas s'attacher exclusivement aux fautes, mais de mettre également en évidence les côtés positifs de la traduction. En effet, la triade faux sens, contresens, non-sens reste unidimensionnelle, parce qu'elle se limite à désigner des erreurs. La valorisation des réussites et la prise en compte des contraintes d'une traduction importent également dans tout processus critique.

\section{RÉFÉRENCES}

Auroux, S. (dir.) (1990): Les notions philosophiques, dictionnaire, Paris, PUF. Benveniste, E. (1974): Problèmes de linguistique générale 2, Paris, Gallimard.

Delisle, J., Lee-Jahnke, H. et M. C. Cormier (1999) : Terminologie de la traduction, Amsterdam/ Philadelphia, John Benjamins.

Drosdowsкi, G. (dir.) (1993): Das große Wörterbuch der deutschen Sprache in acht Bänden, Mannheim-Leipzig-Wien-Zürich, Dudenverlag.

Dubois, J. (dir.) (1979.): Larousse de la langue française, Lexis, Paris.

Dussart, A. (1998) : «Mario Wandruszka ou la stylistique comparée dans une perspective européenne», dans Ballard, M., Europe et traduction, Artois Presses Université/ Les Presses de l'Université d'Ottawa, pp. 323-341.

Guillemin-Flescher, J. (1988) [1981] : Syntaxe comparée du français et de l'anglais, Problèmes de traduction, Gap, Ophrys.

Güttinger, F. (1963): Zielsprache, Theorie und Technik des Übersetzens, Zürich, Manesse Verlag. Halliday, M.A.K. (1978): Language as Social Semiotic, The Social Interpretation of Language and Meaning, London, Arnold.

KADE, O. (1980): Die Sprachmittlung als gesellschaftliche Erscheinung und Gegenstand wissenschaftlicher Untersuchung, Leipzig.

Kundera, M. (1993): Les testaments trahis, essai, Paris, Gallimard.

Ladmiral, J.-R. (1979): Théorèmes pour la traduction, Paris, Payot.

Lalande, A. (1988): Vocabulaire technique et critique de la philosophie, Paris, PUF.

Malblanc, A. (1968): Stylistique comparée du français et de l'allemand, Essai de représentation linguistique comparée et Etude de traduction, Paris, Didier. 
Mossop, B. (2001): Revising and Editing for Translators, Manchester, St Jerome Publishing.

Mounin, G. (1963): Les problèmes théoriques de la traduction, Paris, Gallimard.

Rey, A. et J. Rey-Debove (2001): Le Grand Robert de la langue française, Paris.

Shuttleworth, M. and M. Cowie (1999²) [1997]: Dictionary of Translation Studies, Manchester: St Jerome Publishing.

Trésor de la langue française, Dictionnaire de la langue du $19^{e}$ et du $20^{\circ}$ siècle (1992), Paris/Nancy, Gallimard-CNRS.

VInAY, J.-P. et J. Darbelnet (1978) [1958] : Stylistique comparée du français et de l'anglais, méthode de traduction, Paris, Didier. 